\title{
Theoretical modeling of the electronic structure and exchange interactions in a $\mathrm{Cu}(\mathrm{II}) \mathrm{Pc}$ one-dimensional chain
}

\author{
Wei $\mathrm{Wu}^{1,2}{ }^{*}$ A. J. Fisher ${ }^{2}$, and N. M. Harrison ${ }^{1}$ \\ Department of Chemistry and London Centre for Nanotechnology, \\ Imperial College London, South Kensington Campus, London, SW7 2AZ, U.K. ${ }^{1}$ and \\ UCL Department of Physics and Astronomy and London Centre for Nanotechnology, \\ University College London, Gower Street, London, WC1E 6BT, U.K. ${ }^{2}$
}

\begin{abstract}
We calculate the electronic structure and exchange interactions in a copper(II)phthalocyanine $(\mathrm{Cu}(\mathrm{II}) \mathrm{Pc})$ crystal as a one-dimensional molecular chain using hybrid exchange density functional theory (DFT). In addition, the intermolecular exchange interactions are also calculated in a molecular dimer using Green's function perturbation theory (GFPT) to illustrate the underlying physics. We find that the exchange interactions depend strongly on the stacking angle, but weakly on the sliding angle (defined in the text). The hybrid DFT calculations also provide an insight into the electronic structure of the $\mathrm{Cu}(\mathrm{II}) \mathrm{Pc}$ molecular chain and demonstrate that on-site electron correlations have a significant effect on the nature of the ground state, the band gap and magnetic excitations. The exchange interactions predicted by our DFT calculations and GFPT calculations agree qualitatively with the recent experimental results on newly found $\eta-\mathrm{Cu}(\mathrm{II}) \mathrm{Pc}$ and the previous results for the $\alpha$ - and $\beta$-phases. This work provides a reliable theoretical basis for the further application of $\mathrm{Cu}(\mathrm{II}) \mathrm{Pc}$ to molecular spintronics and organic-based quantum information processing.
\end{abstract}

PACS numbers: $75.50 . \mathrm{Xx}, 71.15 . \mathrm{Mb}, 71.70 . \mathrm{Gm}$

\section{INTRODUCTION}

Magnetic organic materials [1-5] combining optical and magnetic functionality are promising candidates for novel spintronical devices [5-8] and quantum information processing (QIP) $[9,10]$. The electronic structure of magnetic organic materials is also of fundamental interest and underlies both their magnetic and optical properties. The exchange interactions between molecular units largely determine the Neel temperature $\left(T_{N}\right)$ or Curie temperature $\left(\mathrm{T}_{\mathrm{C}}\right)$ for the transition from paramagnetic to ordered anti-ferromagnetic (AFM) or ferromagnetic (FM) phases. The theoretical prediction of the band gap of materials is particularly important for the understanding of optical properties.

Transition-metal phthalocyanines (TMPcs) are well known organic semiconductors [11] that can carry ions with unpaired electron spins in their molecular centres, e.g., copper(II)phthalocyanine $\left(\mathrm{Cu}(\mathrm{II}) \mathrm{Pc}\right.$, spin- $\left.\frac{1}{2}\right)$. In previous work we have reported the electronic structure of the $\mathrm{Cu}(\mathrm{II}) \mathrm{Pc}$ molecule and the variation of exchange interactions as a function of the molecular stacking angle in a molecular dimer $[10,12]$. The calculations of the electronic structure of particular crystalline polymorphs of $\mathrm{Cu}(\mathrm{II}) \mathrm{Pc}$ have previously been reported by several groups [13-16]. However, in these works the spin structure of the material was not considered in detail, and there was no attempt to calculate the exchange interaction between copper spins; indeed, in [15] the spin nature of the ground state found is not explained and a non-spin-resolved band structure is presented. In ref.[16] it was found that the

*Electronic address: w.wu@imperial.ac.uk highest occupied state is of $b_{1 g}$ symmetry and derived from a $\mathrm{Cu}$ d-state based on pure DFT. Using a DFT $+\mathrm{U}$ treatment of the local correlations at the $\mathrm{Cu}$ site, the band gap was computed to be $1.5 \mathrm{eV}$ and the splitting between the majority and minority spin states of the $\mathrm{Cu}$ was $2 \mathrm{eV}$ [16].

The dominant exchange interactions occur within molecular chains along the stacking axis. The stacking angle and the sliding angle (defined in §III) together determine the stacking axis. The magnetic interactions between chains are very weak and presumably dipolar in origin, owing to the large distance $(>10 \AA)$ between copper atoms in the neighbouring chains. The dominant exchange interactions in a $\mathrm{Cu}(\mathrm{II}) \mathrm{Pc}$ crystal may therefore be modeled using a one-dimensional molecular chain.

Recently a new stable form of $\mathrm{Cu}(\mathrm{II}) \mathrm{Pc}$ was reported, known as the $\eta$-phase [10] and possessing a wire-like morphology. Unlike the previously known $\alpha$ and $\beta$ phases, the $\eta$-phase $\mathrm{Cu}(\mathrm{II}) \mathrm{Pc}$ displays a sliding angle significantly different from the plus phase and the cross phase as defined in ref.[12], implying the effect of the sliding angle as a structural parameter needs to be carefully considered. The possibility of the variation of the sliding angle opens up a very wide range of potential structures that can be prepared with comparable stability; indeed, many such structures are already known but their magnetic properties are not yet determined $[17,18]$. Our aim in this paper is to assess systematically the importance of sliding angle for the magnetic interactions, and in doing so to provide a theoretical reference for the geometries, electronic structure, and magnetic properties of new morphologies of $\mathrm{Cu}(\mathrm{II}) \mathrm{Pc}$ (and possibly other transition metal phthalocyanines), which could be found in future experiments. We have therefore modeled the $\mathrm{Cu}(\mathrm{II}) \mathrm{Pc}$ one-dimensional chain for a wide range of stacking and sliding angles. 
Compared with our previous paper [12] we extend our modeling to a larger geometrical parameter space and extend the dimer model to a one-dimensional molecular chain with periodic boundary conditions - thereby isolating the important intra-chain magnetic interactions.

The investigation of the $\mathrm{Cu}(\mathrm{II}) \mathrm{Pc}$ one-dimensional chain falls into four parts. In $\S$ II we give the details of the computational methods and software. In $\S I I I$ we describe the important geometrical parameters, and in particular define the stacking and sliding angles. In $\S I V$ we present the exchange interactions calculated by density functional theory (DFT) and Green's function perturbation theory (GFPT), describe the electronic structure of the $\alpha$-phase of $\mathrm{Cu}$ (II)Pc as a typical example, and discuss these results. Finally in $\S \mathrm{V}$ we draw more general conclusions.

\section{COMPUTATIONAL DETAILS}

We carry out calculations for the $\mathrm{Cu}(\mathrm{II}) \mathrm{Pc}$ single molecule with a $6-31 \mathrm{G}$ basis set [19] in the Gaussian 03 code [20]. The total energies of molecular periodic chains were also calculated using DFT as implemented in the CRYSTAL 06 code [21]. The $6-31 \mathrm{G}$ basis set is designed for use in molecular studies and is inadequate to describe atomic orbitals of the copper atoms in the molecular crystal environment. In order to improve the description, we use a 6-311G Gaussian basis set to describe the atomic orbitals of the copper atoms. The outer $\mathrm{d}$-shell gaussian function of each copper atom, with exponent $0.67 a_{0}^{-2}$, is supplemented by a diffusion gaussian function with an exponent at $0.27 a_{0}^{-2}$ which is between one third and one half of the outer exponent. This successfully enhances the basis set while avoiding pseudo linear dependence. We use the standard molecular 6$31 \mathrm{G}$ basis set for other elements. The Monkhorst-Pack sampling [22] of reciprocal space is carried out choosing a grid of shrinking factor equal to eight. The truncation of the Coulomb and exchange series in direct space is controlled by setting the Gaussian overlap tolerance criteria to $10^{-6}, 10^{-6}, 10^{-6}, 10^{-6}$, and $10^{-12}$ [21]. The self-consistent field (SCF) procedure is converged to a tolerance of $10^{-6}$ a.u. per unit cell (p.u.c). To accelerate convergence of the SCF process, all calculations have been performed adopting a linear mixing of Fock matrices by 30 percent.

Electronic exchange and correlation are described using the B3LYP hybrid functional [23]. The advantages of B3LYP include a partial elimination of the selfinteraction error and balancing the tendencies to delocalize and localize wave-functions by mixing Fock exchange with that from a generalized gradient approximation (GGA) exchange functional [23]. The brokensymmetry method [24] is used to localize opposite electron spins on each molecule in order to describe the AFM state. The exchange coupling, $J$ in the one-dimensional
Heisenberg spin chain [25] is defined here as,

$$
\hat{H}=2 J \sum_{i} \hat{\vec{S}}_{i} \cdot \hat{\vec{S}}_{i+1},
$$

and determined by

$$
J=\left(E_{\mathrm{FM}}-E_{\mathrm{AFM}}\right) / 2,
$$

where $E_{\mathrm{AFM}}$ and $E_{\mathrm{FM}}$ are the total energies of a supercell containing two molecules in which the spin configurations are anti-aligned and aligned respectively. DFT total-energy calculations have some intrinsic disadvantages for computing exchange interactions, e.g., a comparison between two very large numbers to get a small exchange splitting in an all-electron local basis set formalism. However, the performance of B3LYP as implemented in CRYSTAL has previously been shown to provide an accurate description of the electronic structure and magnetic properties for both inorganic and organic compounds [12, 26-28].

In addition to total energy calculations we use GFPT to compute exchange interaction in a $\mathrm{Cu}(\mathrm{II}) \mathrm{Pc}$ dimer in order to have a qualitative picture of the interaction mechanism. This has the advantage of avoiding the calculation of very small differences between large energies, at the cost of assuming a particular model for the exchange interaction. We adopt the formalism of indirect exchange in a perturbative way as described in the previous work [12]. Here we use Gaussian 03 to compute the single-molecule electronic structure and the core Hamiltonian of a molecular dimer. The perturbative approximation to the exchange interaction is then computed as,

$$
J=A\left(t_{x}^{e} t_{x}^{h}+t_{y}^{e} t_{y}^{h}\right),
$$

where $t_{x, y}^{e}\left(t_{x, y}^{h}\right)$ is the electron (hole) hopping through the lowest unoccupied (highest occupied) $e_{g x}$ and $e_{g y}$ orbitals, and $A$ is an intra-molecular parameter proportional to $\frac{J_{p}^{2}}{U^{3}}$ where $J_{p}$ is the two-electron integral responsible for spin-polarization of ring states by the $\mathrm{Cu}$ spin, and $U$ is the on-site Coulomb interaction. The magnitude of $A$ is irrelevant if we are only interested in the trend of the exchange interaction in the variation of the geometry. In our perturbation theory calculations we therefore only compute the hopping integrals in eq.(3).

The three models adopted here (DFT calculations of a molecular dimer [12] and of a one-dimensional molecular chain, and GFPT calculations for a molecular dimer) provide complementary information about exchange interactions. The first two are used to analyze the electronic structure and to obtain a quantitative description of exchange interactions while the third reveals most clearly the physical mechanism responsible for the exchange interactions.

\section{MOLECULAR GEOMETRY}

The intra-molecular coordinates were determined by the optimization of an isolated $\mathrm{Cu}(\mathrm{II}) \mathrm{Pc}$ molecule. TMPc 
crystals are known to consist of parallel molecular planes along the stacking axis connecting copper atoms. In all our calculations we therefore assume that each molecule is in the same orientation as its neighbours along the molecular chain, with a constant inter-plane separation $d$. Let $\hat{\vec{r}}$ be the projection into the molecular plane of the vector joining the central $\mathrm{Cu}$ atoms of the two molecules. Then we define the stacking angle $\phi$ as the angle between the molecular plane and the stacking axis connecting the copper atoms, and the sliding angle $\psi$ as the angle between $\hat{\vec{r}}$ and the $X$ axis as shown in Fig.1. The $X$ and $Y$ axes are defined within a given molecular plane. Various crystal structures can therefore be generated by varying the inter-plane distance $d$, the stacking angle $\phi$, and the sliding angle $\psi$. These two angles are related to the cartesian coordinates $X$ and $Y$ as $X=d \cot \phi \cos \psi$ and $Y=d \cot \phi \sin \psi$ (Fig. 1$)$.

It would be necessary to have a reliable description of Van der Waals forces to calculate the inter-plane distance $d$ from first principles. However, widely used exchangecorrelation functionals, including B3LYP, do not describe Van der Waals forces reliably. In the following calculations the inter-plane distance $d$ is fixed at the experimentally determined value of $3.42 \AA$, obtained by X-ray diffraction (XRD) of $\mathrm{Cu}(\mathrm{II}) \mathrm{Pc}$ thin films [10, 29]. Calculations have been performed for stacking angles from $20^{\circ}$ to $90^{\circ}$ and sliding angles from $0^{\circ}$ to $45^{\circ}$ with $5^{\circ}$ increments exploiting the $\mathrm{D}_{4 h}$ symmetry of the $\mathrm{Cu}(\mathrm{II}) \mathrm{Pc}$ molecule. So far three phases of $\mathrm{Cu}$ (II)Pc molecular crystals have received extensive experimental study: the $\alpha$ phase with stacking angle $\phi \simeq 63^{\circ}$ and sliding angle $\psi \simeq 7^{\circ}$, the $\beta$-phase with stacking angle $\phi \simeq 45^{\circ}$ and sliding angle $\psi \simeq 43^{\circ}$, and the $\eta$-phase with stacking angle $\phi \simeq 63^{\circ}$ and sliding angle $\psi \simeq 27^{\circ}[10]$.

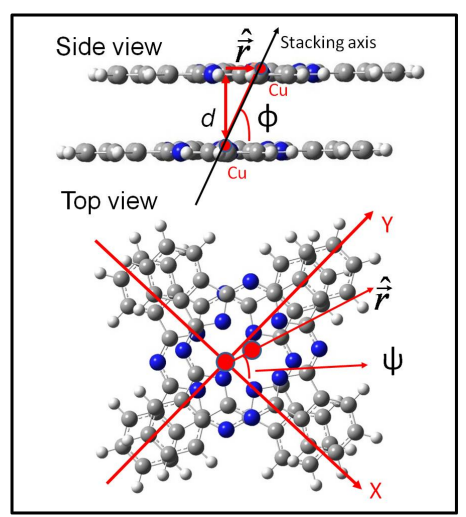

FIG. 1: (Color online.) Two neighboring $\mathrm{Cu}(\mathrm{II}) \mathrm{Pc}$ molecules from a side view and a top view to illustrate important geometrical parameters in the crystal structure. The atoms are color-coded, i.e., copper in red, carbon in grey, nitrogen in blue, and hydrogen in white. The inter-plane distance is labeled as $d$, the stacking angle $\phi$, and the sliding angle $\psi$. The stacking axis is indicated by a black arrow connecting copper atoms. The vector $\vec{r}$, and the $\mathrm{X}$ and $\mathrm{Y}$ axes are used to define the sliding angle, i.e., $\cos \psi=\hat{\vec{r}} \cdot \hat{\vec{X}}$.

\section{RESULTS AND DISCUSSIONS}

\section{A. DFT calculations}

In Fig.(2) the exchange interaction calculated using DFT in a one-dimensional $\mathrm{Cu}(\mathrm{II}) \mathrm{Pc}$ chain is shown as a function of $X$ and $Y$. The exchange interaction depends strongly on stacking angle (distance from the centre of the plot) but weakly on sliding angle. The exchange interaction increases with the stacking angle in the chosen angular region and peaks with a value of $4.78 \mathrm{~K}$ at a stacking angle of $90^{\circ}$.

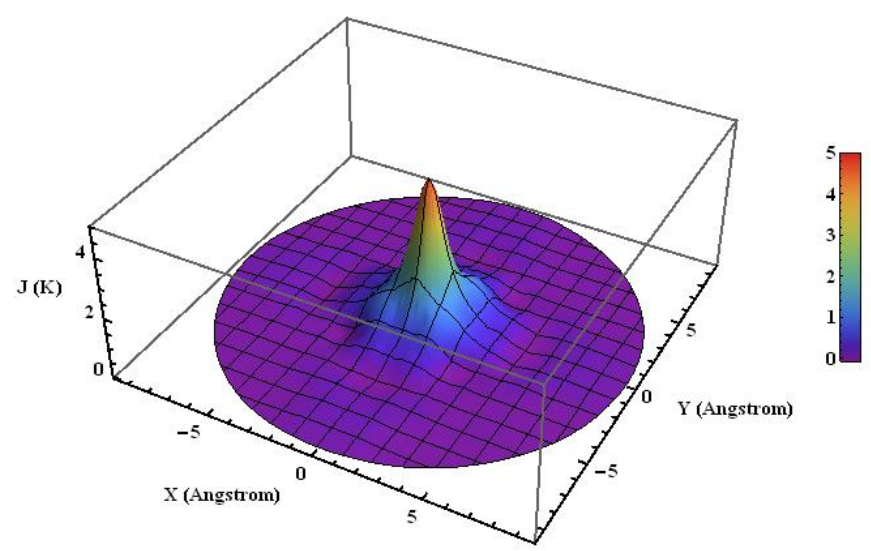

FIG. 2: (Color online.) The exchange interaction calculated using DFT as a function of the cartesian coordinates $X$ and $Y$. Notice that exchange interactions strongly depend on stacking angles (and hence on the distance from the centre of the plot), but weakly on sliding angles, and peak at a stacking angle of $90^{\circ}$ with $4.78 \mathrm{~K}$.

The exchange interactions at the experimentally observed geometries [10] are $J_{\alpha}^{\mathrm{DFT}}=1.30 \mathrm{~K}, J_{\beta}^{\mathrm{DFT}}=$ $0.14 \mathrm{~K}$, and $J_{\eta}^{\mathrm{DFT}}=1.26 \mathrm{~K}$. These results are in qualitative agreement with magnetic measurements using superconducting quantum interference device (SQUID) magnetometry $[3,10]$, from which it has been deduced that $J_{\alpha}^{\exp }=1.4 \mathrm{~K}, J_{\beta}^{\text {exp }}=-0.15 \mathrm{~K}$, and $J_{\eta}^{\exp }=0.5 \mathrm{~K}$. There is a quantitative agreement of the calculated result with the observed result for the $\alpha$-phase, while the $\beta$-pase is paramagnetic $(J \simeq 0)$ within the accuracy of DFT calculations, and also within the experimental uncertainty. There is some discrepancy in the $\eta$-phase, for which only single measurement is available [10]. We expect that basis set errors will largely cancel out when subtracting the energies in equation (2), leaving intrinsic limitations of hybrid DFT as the main source of error. The magnitude of exchange interactions is in the order of $1 \mathrm{~K}$; this is much less then the DFT error in a single total energy, but it has been shown previously that the cancelation of errors in states differing just in their spin orientations can yield exchange constants in hybrid DFT accurate to about $1 \mathrm{~K}$-about 9 orders of magnitude smaller than the individual total energies. In this context the agreement of the calculations with the experiments is seen to 
be remarkably good. These calculations also agree qualitatively with the results of the previous DFT calculations for the molecular dimers in these three phases $[10,12]$.

The total energy per unit cell of the $\alpha$-phase is approximately $2 \mathrm{meV}$ higher than that of the $\eta$-phase, and the total energies of both of them are much higher than the $\beta$-phase by $0.4 \mathrm{eV}$. This suggests the $\pi-\pi$ electronic repulsion between rings might be important in the molecular interaction in $\mathrm{Cu}(\mathrm{II}) \mathrm{Pc}$ though Van der Waals forces also play a crucial role and are not fully accounted for in our calculations.

The electronic structures of the $\alpha$-, the $\beta$-, and the $\eta$ phases are shown in Fig.(3), Fig.(4), and Fig.(5), respectively. In the band structures of all these three phases, $k_{x}$ is oriented along the stacking axis of the molecular chain and the zero of energy for the DOS plots is chosen to be at mid-gap. 21 occupied and 10 unoccupied bands are plotted in the band structure from $k_{x}=0$ to $k_{x}=2 \pi / a$ with $a=9.56 \AA$ in the AFM configurations (Fig.3-5(a)), while 22 (20) occupied bands and 9 (11) unoccupied bands of spin-up (spin-down) are plotted in the FM configurations (Fig.3-5(d)). The corresponding densities of states (DOS) are shown for the AFM (Fig.3-5(b)) and FM configurations (Fig.3-5(e)). Two valence bands are derived from the highest occupied molecular orbitals (HOMO), and four conduction bands from the lowest unoccupied molecular orbtals (LUMO) respectively of the $\mathrm{Cu}$ (II)Pc molecule. The band gap is about $2.0 \mathrm{eV}$ and occurs between $\pi$ and $\pi^{*}$ states of the Pc ring. The band gap is significantly increased by our hybrid DFT calculations compared with the previous work in which on-site electron correlation is neglected [13-15], where the gap is significantly smaller (only about $1 \mathrm{eV}$ ). Furthermore in several previous calculations the lowest unoccupied state is a $\mathrm{Cu}$ d-state, in contrast to the molecular HOMO as we find. It is interesting that when on-site electron correlation is taken into account empirically at the $\mathrm{DFT}+\mathrm{U}$ level with an on-site $U$ about $5 \mathrm{eV}$ [16], the band gap is opened up but by somewhat less than in hybrid DFT (to approximately $1.5 \mathrm{eV}$ ). Optical absorption measurements for $\mathrm{Cu}(\mathrm{II}) \mathrm{Pc}$ crystals [11] show the wavelength of the $\mathrm{Q}$ absorption band arising from the HOMO-LUMO transition is about $650 \mathrm{~nm}(\simeq 1.9 \mathrm{eV})$, in a good agreement with the band gap in our calculations.

The projected DOS (PDOS) onto two copper atoms in a supercell is also plotted along with the total DOS, colour-coded consistent with that in the spin densities, to illustrate the occupancy of the atomic orbitals on the copper atoms. The spin densities for the AFM configuration (FM configuration) are shown in Fig.3-5(c) (Fig.3-5(f)), respectively. The singly occupied bands can be found by comparing the projected density of states of the AFM and FM configurations. There are two peaks in the projected DOS on the left of the valence bands in spin-up and spin-down in AFM configuration (Fig.3-5(b)) while there are two peaks in the same energy position only in spin-up in the FM configuration (Fig.3-5(e)). So these peaks correspond to the singly occupied narrow bands.
The spin densities of AFM (Fig.3-5(c)) and FM (Fig.3$5(\mathrm{f})$ ) configurations both indicate the singly occupied orbital is $d_{x^{2}-y^{2}}$; this is in agreement with the results of our previous DFT calculations for the $\mathrm{Cu}(\mathrm{II}) \mathrm{Pc}$ single molecule and dimer. The Mulliken charges on the copper atoms are about $+0.9|e|$ for the AFM and FM configurations, and the Mulliken magnetic moments on two copper atoms for AFM $(\mathrm{FM})$ are about $0.65 \mu_{B}\left(0.65 \mu_{B}\right)$ and $-0.65 \mu_{B}\left(0.65 \mu_{B}\right)$, respectively.

The electron hopping integral between copper atoms is very small as suggested by the small bandwidth of these singly occupied bands, and this further indicates that it is difficult for copper spins to interact directly. In particular, the narrow bandwidth suggests that the superexchange mechanism as defined in Ref. [31] will be suppressed, since it relies on virtual charge excitations of the $\mathrm{Cu}$ sites. However, the singly occupied band is energetically very close to a set of ligand bands, and spin polarization of the ligand due to copper spins is facile; this opens up the possibility of indirect exchange between the $\mathrm{Cu}$ sites involving polarization of the surrounding ligand spins. The gap between the upper and lower Hubbard bands is easily identified as about $4 \mathrm{eV}$ in both the band structure and the DOS; the significantly greater splitting compared with the previous work [13-15] drives both the filled and empty d-states away from the Fermi energy, in qualitative agreement with $\mathrm{DFT}+\mathrm{U}$ calculations [16].

The electronic structures of these three phases share many qualitative features in terms of the orbital occupancy, the band gap, the on-site Coulomb interaction on the copper site, etc as shown in Fig. (3-5). However, the transfer integrals are sensitive to the local geometry and thus the bandwidth varies significantly as the geometry changes. For example, LUMO bandwidths of $\alpha$-phase $(\sim 0.5 \mathrm{eV}), \eta$-phase $(\sim 0.3 \mathrm{eV})$ and $\beta$-phase $(\sim 0.2 \mathrm{eV})$ differ significantly. This reflects the significant variation in the exchange; using perturbation theory, we now make this connection more directly.

\section{B. Perturbation theory calculations}

The variation of the exchange interactions in a dimer for the same set of geometrical parameters used in the DFT calculations has been estimated using GFPT, based on the model; of indirect exchange [30, 31] . The exchange interactions are once again strongly dependent on the stacking angle but weakly on the sliding angle, as in the DFT calculations for one-dimensional chain. The exchange interaction using GFPT peaks at a stacking angle $90^{\circ}$ and is 12 times larger than that in the $\alpha$-phase. The ratio of the exchange interaction of $\eta$-phase to $\alpha$-phase is 0.4 in agreement with that observed [10]. In fact this GFPT result more closely resembles the trend observed experimentally than the DFT result for which the ratio is about 1. Furthermore, the oscillating behavior of the exchange interactions with the stacking angle is more obvious in perturbation theory than the DFT calculations. 
$\alpha$-phase

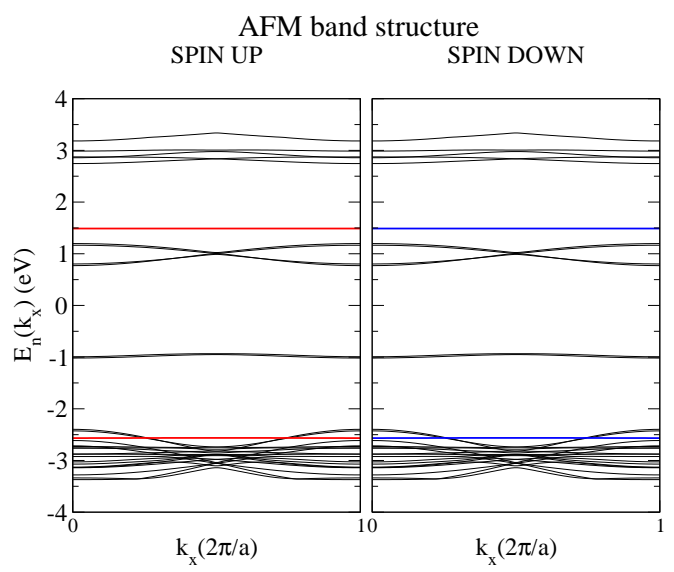

(a)

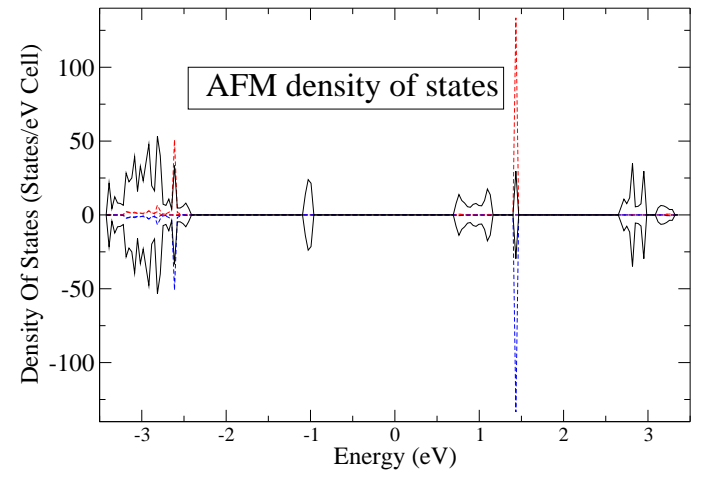

(b)

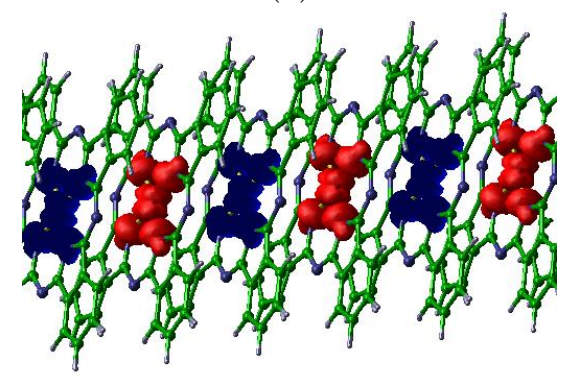

(c)

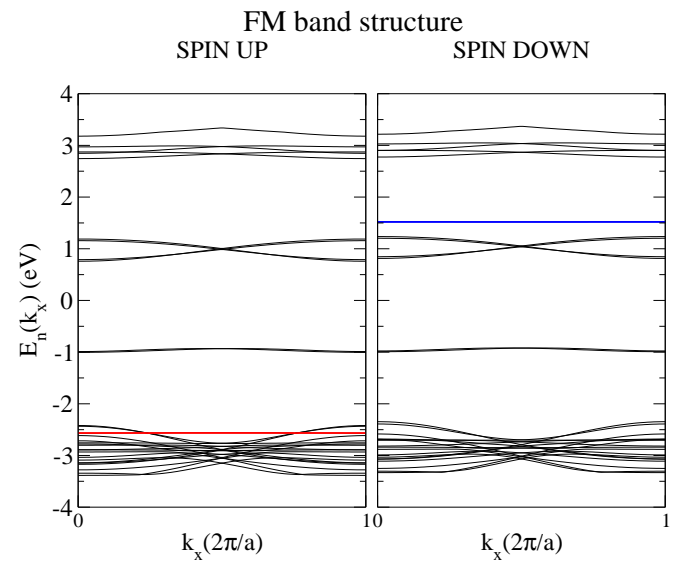

(d)

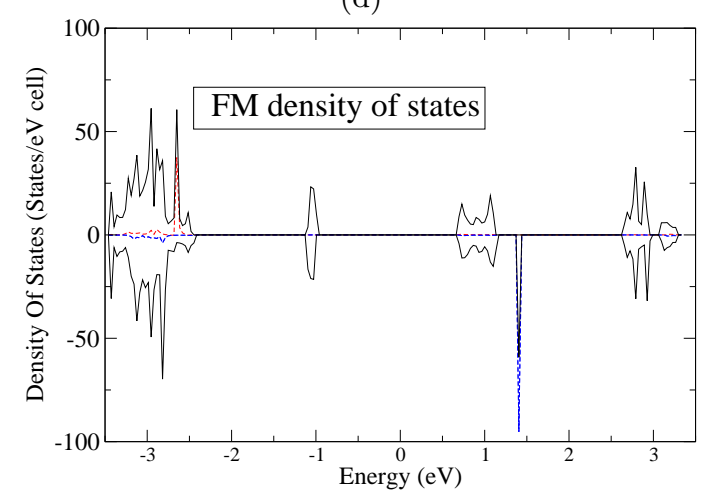

(e)

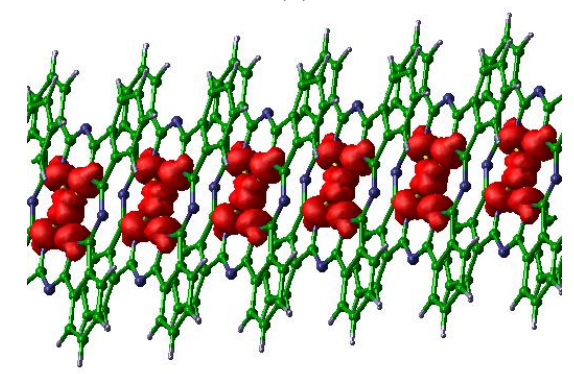

(f)

FIG. 3: (Color online.) The band structure, density of states, and spin densities of AFM (left column) and FM (right column) configurations for $\alpha$-phase $\mathrm{Cu}(\mathrm{II}) \mathrm{Pc}$ are shown. In band structure the d-bands in spin-up are highlighted in red, and spin-down in blue. Notice that these bands are very narrow. In the density of states the spin-up is displayed as positive and spin-down as negative, and the projected density of states is scaled by a factor of 5.0 to illustrate the singly occupied orbitals. The spin densities show the spin configurations in different calculations where spin up is in red and spin down in blue.

These comparisons suggest that GFPT based on indirect exchange indeed captures the dominant physical mechanism of the exchange interactions.

\section{CONCLUSIONS}

We have calculated the exchange interaction in $\mathrm{Cu}(\mathrm{II}) \mathrm{Pc}$ for a variety of sliding and stacking angles which include the observed $\alpha$-, $\beta$-, and $\eta$-phases both us- ing DFT for one-dimension molecular chains and GFPT for molecular dimers. The qualitative trends of exchange interactions predicted by DFT and GFPT agree with those observed experimentally. Exchange interactions computed using DFT and GFPT are strongly dependent on stacking angles, but only weakly on sliding angles.

The DFT calculations give insight into the underlying electronic structure of the $\mathrm{Cu}(\mathrm{II}) \mathrm{Pc}$ molecule chain and its relationship to the magnetic properties. Valence and conduction bandwidths, the band gap, and the DOS 
$\beta$-phase

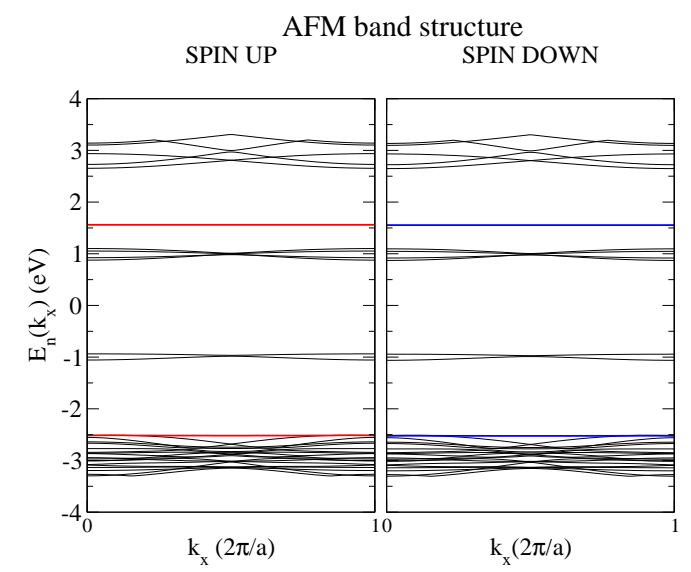

(a)

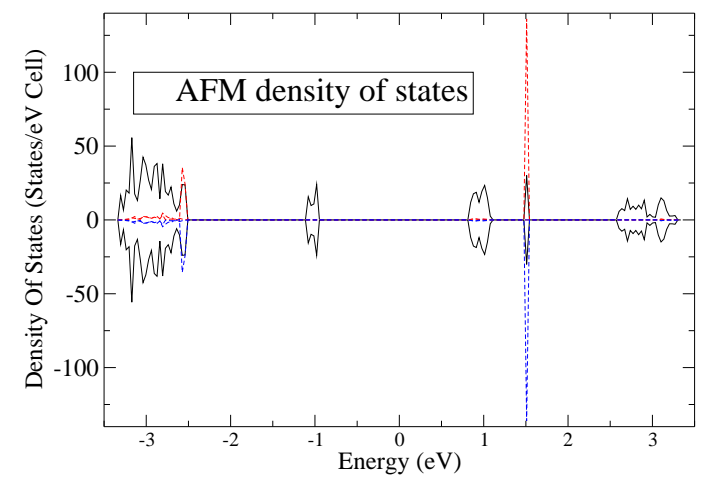

(b)

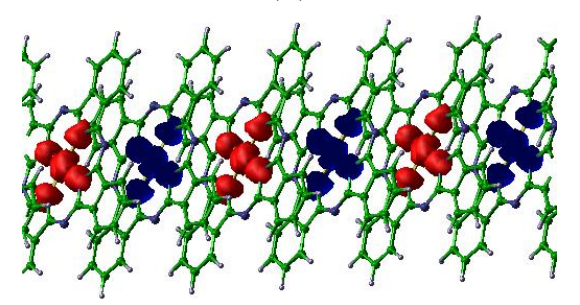

(c)

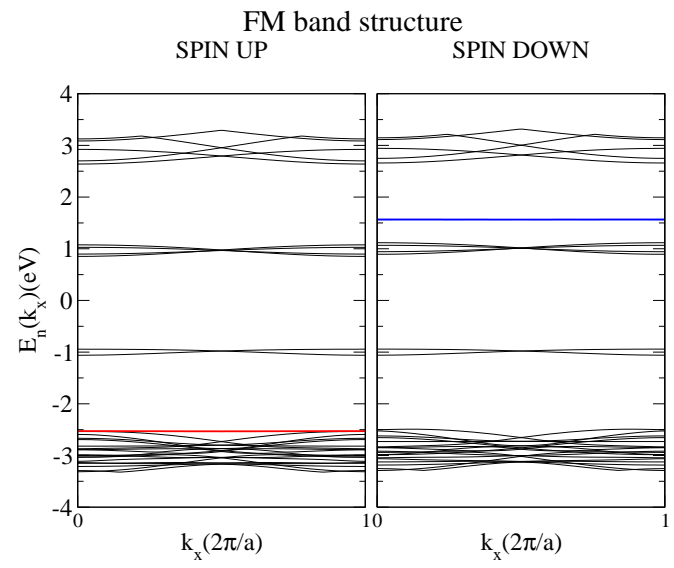

(d)

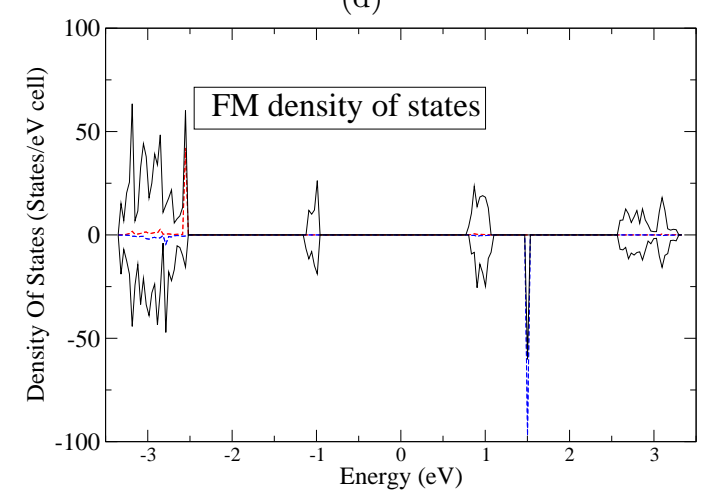

(e)

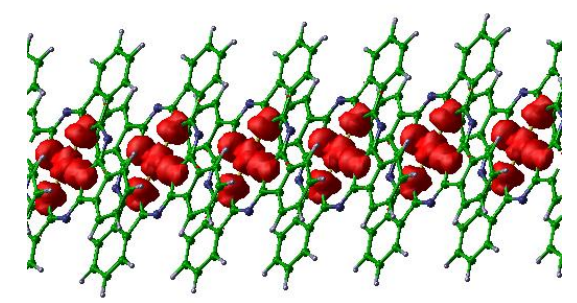

(f)

FIG. 4: (Color online.) The band structure, density of states, and spin densities of AFM (left column) and FM (right column) configurations for $\beta$-phase $\mathrm{Cu}(\mathrm{II}) \mathrm{Pc}$ are shown. In band structure the d-bands in spin-up are highlighted in red, and spin-down in blue. Notice that these bands are very narrow. In the density of states the spin-up is displayed as positive and spin-down as negative, and the projected density of states is scaled by a factor of 5.0 to illustrate the singly occupied orbitals. The spin densities show the spin configurations in different calculations where spin up is in red and spin down in blue.

are all important for understanding various optical and transport measurements in $\mathrm{Cu}(\mathrm{II}) \mathrm{Pc}$. We find that the band gaps and d-d splittings are predicted to be significantly larger than in previous work [13-15] thanks to the use of the hybrid exchange density functional, leading to an improved description of the on-site correlation [16]. The DOS in our calculations is in qualitative agreement with the optical experiments shown in [10]. Our calculations have therefore shown a significant improvement in the treatment of the electronic structure of $\mathrm{Cu}(\mathrm{II}) \mathrm{Pc}$ crystals, without the need to introduce any materialspecific parameters.
In particular the variation of the valence and conduction bandwidths between structures drives the computed (and experimentally observed) variation in the exchange constants. One important consequence is that, at least for this type of interaction mechanism, synthetic strategies aimed at maximizing the transition temperature should seek stacking angles as close to $90^{\circ}$ as possible; another is that such geometries are also likely to give the highest charge-transport mobilities [32].

An important technical feature of our DFT calculations, employing a hybrid exchange-correlation functional, is the ability to describe localized unpaired d- 
$\eta$-phase

AFM band structure SPIN UP

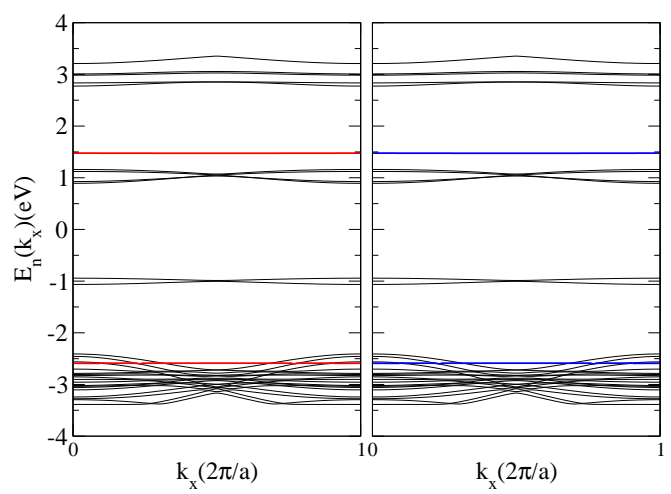

(a)

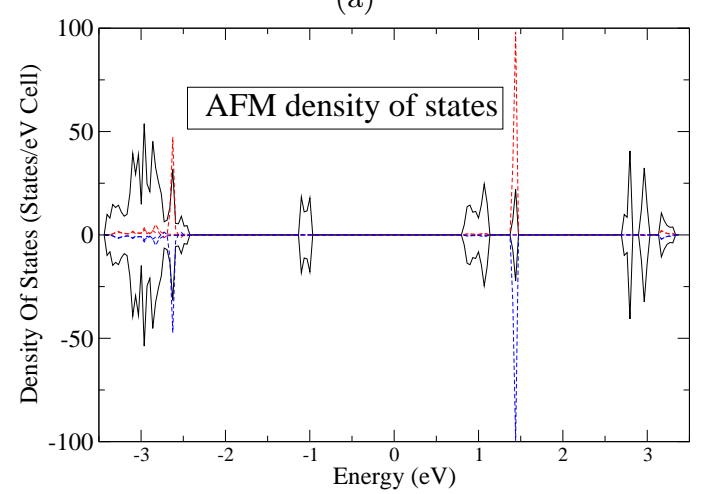

(b)

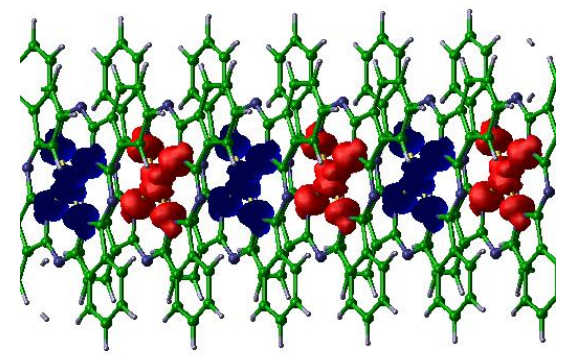

(c)
FM band structure SPIN UP

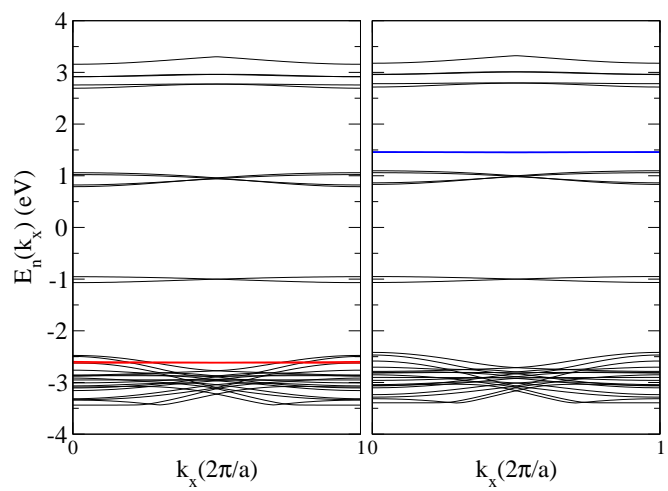

(d)

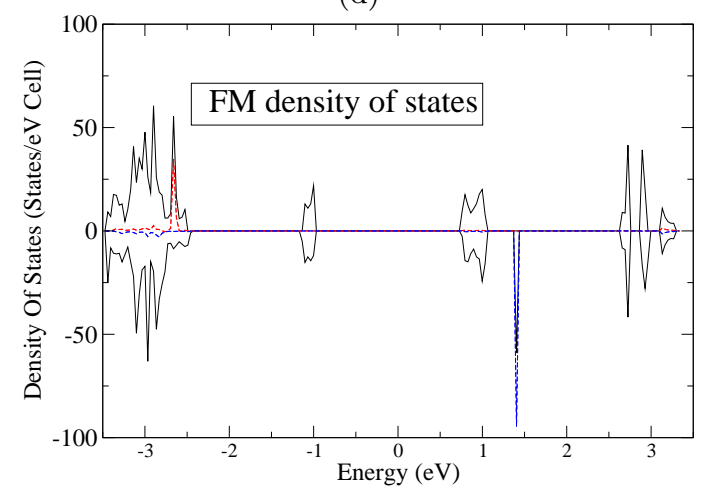

(e)

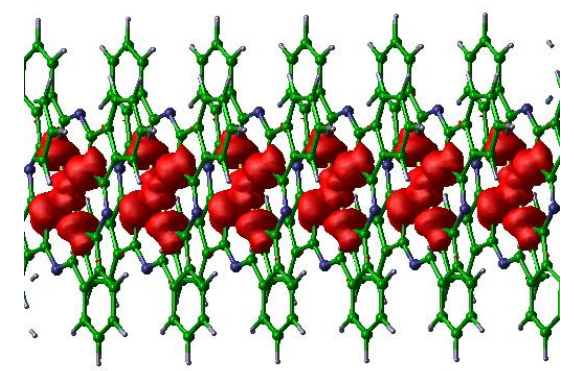

(f)

FIG. 5: (Color online.) The band structure, density of states, and spin densities of AFM (left column) and FM (right column) configurations for $\eta$-phase $\mathrm{Cu}(\mathrm{II}) \mathrm{Pc}$ are shown. In band structure the d-bands in spin-up are highlighted in red, and spin-down in blue. Notice that these bands are very narrow. In the density of states the spin-up is displayed as positive and spin-down as negative, and the projected density of states is scaled by a factor of 5.0 to illustrate the singly occupied orbitals. The spin densities show the spin configurations in different calculations where spin up is in red and spin down in blue.

electron and delocalized ligand states simultaneously; the $\mathrm{DFT}+\mathrm{U}$ approach $[16,33]$ with a plane-wave basis, incorporates similar physics gives similar results provided $U$ is chosen appropriately. A detailed comparison will be presented in a forthcoming paper [34]. Calculations of both types therefore provide a valuable basis for the further theoretical investigation of devices combining optics and magnetism or electronics and magnetism, e.g., magneto-resistant devices.

As shown by the GFPT results, the dominant mag- netic interaction mechanism in $\mathrm{Cu}(\mathrm{II}) \mathrm{Pc}$ is indirect exchange $[30,31]$ which arises from the spin polarization of the ligand due to the central unpaired copper spin and the consequent delocalization of this spin polarization through the chain of delocalized $\pi$-conjugated ring systems formed by the stacked $\mathrm{Cu}(\mathrm{II}) \mathrm{Pc}$ molecules. Similar delocalization due to $\pi$-conjugation occurs in other organic systems, most notably in graphene [35]; it is therefore not surprising that the connection between spin and charge transport implied by our results has been noticed 


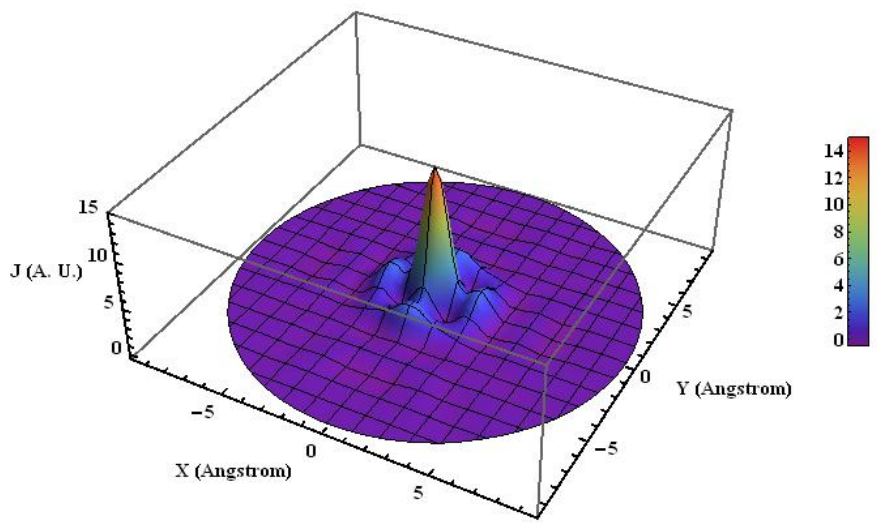

FIG. 6: (Color online.) Exchange interactions of $\mathrm{Cu}(\mathrm{II}) \mathrm{Pc}$ dimer calculated using perturbation theory as a function of the cartesian coordinates $X$ and $Y$. Notice that exchange interactions strongly depend on stacking angles, but weakly on sliding angles. in other systems as far back as the early 1990s, where triplet energy transfer was attributed to double electron exchange [36] in a similar way to our work.

\section{Acknowledgments}

We wish to acknowledge the support of the UK Research Councils Basic Technology Programme under grant EP/F041349/1. We thank Gabriel Aeppli, Salahud Din, Sandrine Heutz, Chris Kay, Soumaya Mauthoor, Marshall Stoneham, Hai Wang, and Marc Warner for helpful discussions.
[1] Joel S. Miller, Arthur J. Epstein, William M. Reiff, Science, 240, 40 (2000).

[2] James C. Ellenbogen and Kwan S. Kwok, Materials Today, 5, 28 (2002).

[3] S. Heutz, C. Mitra, Wei Wu, A. J. Fisher, A. Kerridge, A.M. Stoneham, A. H. Harker, J. Gardener, H.-H. Tseng, T. S. Jones, C. Renner, and G. Aeppli, Adv. Mat., 19, 3618 (2007).

[4] S. Pramanik, C.-G. Stefanita, S. Patibandla, S. Bandyopadhyay, K. Garre, N. Harth and M. Cahay, Nat. Nano., 2216 (2007).

[5] Z. H. Xiong, Di Wu, Z. Valy Vardeny, and Jing Shi, Nature, 427, 821 (2004).

[6] V.N. Prigodin, N.P. Raju, K.I. Pokhodnya, J.S. Miller and A.J. Epstein, Adv. Mat., 14, 1230 (2002).

[7] Stefano Sanvito, Nat. Mat. 6, 803 (2007).

[8] Jung-Woo Yoo, Chia-Yi Chen, H.W. Jang, C.W. Bark, V. N. Prigodin, C. B. Eom, and A. J. Epstein, Nat. Mat., 9, 638 (2010).

[9] Grigore A. Timco, Stefano Carretta, Filippo Troiani, Floriana Tuna, Robin J. Pritchard, Christopher A. Muryn, Eric J. L. McInnes, Alberto Ghirri, Andrea Candini, Paolo Santini, Giuseppe Amoretti, Marco Affronte, and Richard E. P. Winpenny, Nat. Nano. 4, 173 (2009).

[10] Hai Wang, Soumaya Mauthoor, Salahud Din, Jules A. Gardener, Rio Chang, Marc Warner, Gabriel Aeppli, David W. McComb, Mary P. Ryan, Wei Wu, Andrew J. Fisher, Marshall Stoneham and Sandrine Heutz, ACS Nano, 4, 3921 (2010).

[11] The porphyrins edited by David Dolphin, Academic Press, 1979

[12] Wei Wu, A. Kerridge, A. H. Harker, and A. J. Fisher, Phy. Rev. B 77, 184403 (2008).

[13] B. Bialek, In Gee Kim, and Jae Il Lee, Thin Solid Films 436, 107 (2003).

[14] L. Lozzi, S. Santucci, S. La Rosa, B. Delley, and S. Picozzi, J. Chem. Phys. 121, 1883 (2004).

[15] Yanting Yang, Yanming Yang, Fugen $\mathrm{Wu}$, and Zhigang Wei, Solid State Communications 148, 559 (2008).
[16] Gianluca Giovannetti, Geert Brocks, and Jeroen van den Brink, Phys. Rev. B 77,035133 (2008).

[17] Peter Erk, Current Opinion in Solid State and Materials Science, 5, 155 (2001).

[18] Peter Erk, Heidi Hengelsberg, Mairi F. Haddow and Richard van Gelder, Cryst. Eng. Comm., 6, 474 (2004).

[19] R. Ditchfield, W. J. Hehre, and J. A. Pople, J. Chem. Phys., 54724 (1971).

[20] M. J. Frisch, et al., Gaussian 03 (Gaussian, Inc., Pittsburgh, PA, 1998).

[21] R. Dovesi, V. R. Saunders, R. Roetti, R. Orlando, C. M. Zicovich-Wilson, F. Pascale, B. Civalleri, K. Doll, N. M. Harrison, I. J. Bush, P. DArco, and M. Llunell, CRYSTAL06 (CRYSTAL06 User's Manual. University of Torino, Torino, 2006).

[22] H. J. Monkhorst and J. D. Pack, Phys. Rev. B 13, 5188 (1976).

[23] A. D. Becke, J. Chem. Phys. 98, 5648 (1993).

[24] L. Noodleman, J. Chem. Phys. 74, 5737 (1980).

[25] W. Heisenberg, Z. Phys. 49, 619 (1928).

[26] F. Illas, I de P.R. Moreira, C. de Graaf and V. Barone, Theo. Chem. Acc, 104, 265 (2000).

[27] J. Muscata, A. Wanderb, and N.M. Harrison, Chem. Phys. Lett. 342, 397 (2001).

[28] Ling Ge, Barbara Montanari, John H. Jefferson, David G. Pettifor, Nicholas M. Harrison, and G. Andrew D. Briggs, Phys. Rev. B 77, 235416 (2008).

[29] A. Hoshino, Y. Takenaka and H. Miyaji, Acta Cryst. B59, 393 (2003).

[30] M. A. Ruderman and C. Kittel, Phys. Rev. 96, 99 (1954).

[31] P. W. Anderson, Phys, Rev, 115, 2 (1959).

[32] W.J. Pietro, T.J. Marks and M.A. Ratner, J. Am. Chem. Soc. 1075387 (1985).

[33] A.I. Liechtenstein, V.I. Anisinov and J. Zaanen, Phys. Rev. B 52 R5467 (1995).

[34] N. Kearsey et. al., in preparation.

[35] A. K. Geim and K. S. Novoselov, Nat. Mat. 6, 183 (2007).

[36] Gerhard L. Closs, Mark D. Johnson, John R. Miller, Piotr Piotrowiak, J. Am. Chem. Soc. 1113751 (1989). 